\title{
Global parameters for characterizing the radial and azimuthal polarization content of totally polarized beams
}

\author{
R. Martínez-Herrero, P.M. Mejías*, G. Piquero, V. Ramírez-Sánchez \\ Departamento de Óptica, Facultad de Ciencias Físicas, Universidad Complutense de Madrid, 28040 Madrid, Spain
}

Received 25 May 2007; received in revised form 4 December 2007; accepted 7 December 2007

\begin{abstract}
Several global parameters are proposed to characterize the radial and azimuthal polarization content of non-uniformly totally polarized beams. Such figures of merit can be written and measured in terms of two Stokes parameters, and also from the data at the output of either a radial or an azimuthal dichroic polarizer, integrated throughout the beam profile. The measurability of the proposed parameters has also been experimentally checked.
\end{abstract}

(C) 2007 Elsevier B.V. All rights reserved.

PACS: 41.85.Ew; 42.25.Ja

Keywords: Beam characterization; Beam polarization; Polarization standards

\section{Introduction}

In recent years, it has been shown that beams with radial or azimuthal polarization can be of use in Optics, as occurs, for example, in high-resolution microscopy, particle trapping, material processing and amplification in strongly pumped solid-state rods (see, for example, Refs. [1-19]). Moreover, radial and azimuthal polarizers have been implemented and are now commercially available (for instance, dichroic and liquid-crystal-based devices). On the other hand, to describe by means of a single number the transverse polarization structure of a beam, it has been defined in the literature the so-called standard degree of polarization of a general beam, $P,[20,21]$ (defined in terms of the Stokes parameters) whose determination procedure involve measurements of the integrated beam irradiance over the full detection area. For non-uniformly totally polarized beams, however, this parameter is not appropriate: note, for instance, that $P$ equals zero for totally radially polarized beams. Consequently, in such cases it

\footnotetext{
${ }^{*}$ Corresponding author.

E-mail address: pmmejias@fis.ucm.es (P.M. Mejías).
}

would be useful to introduce new overall parameters to properly characterize the distribution of the polarization over the cross-sectional area.

Up to now, several global measurable parameters have been proposed for describing non-uniformly totally polarized beams [22-25]. In the present work the attention is focused on the radial and azimuthal polarization content of a totally polarized beam over the regions of its wavefront where the irradiance is not negligible.

The paper is arranged as follows. In the next section, the formalism and the key overall definitions are introduced. In Section 3, the proposed figures of merit are given in terms of the Stokes parameters, and, in the same section, we also show how the new characteristic parameters can be analytical and experimentally obtained from the output of radial and azimuthal dichroic polarizers. In Section 4, the measurability of these parameters is checked and, finally, the main conclusions are summarized in Section 5.

\section{Formalism and key definitions}

Let us consider a quasimonochromatic paraxial beam propagating along the $z$-axis. The transverse electric field 
vector, $\mathbf{E}$, associated to a non-uniformly polarized beam, can be written in planar polar coordinates as

$\mathbf{E}(r, \theta)=\left(\begin{array}{c}E_{s} \\ E_{p}\end{array}\right)=E_{0}(r, \theta)\left(\begin{array}{c}f(r, \theta) \\ g(r, \theta)\end{array}\right)$,

where $s$ and $p$ denote the transverse field components orthogonal to the $z$-axis, $f$ and $g$ are deterministic functions, $E_{0}$ represents an stochastic process (due to random fluctuation of the field) and $r$ and $\theta$ are the polar coordinates. Let $\mathbf{u}_{R}$ and $\mathbf{u}_{\theta}$ be orthogonal unitary vectors in the radial and azimuthal directions, respectively:

$\mathbf{u}_{R}=(\cos \theta, \sin \theta)$,

$\mathbf{u}_{\theta}=(-\sin \theta, \cos \theta)$.

In terms of these vectors, the electric field $\mathbf{E}$ can be written in the form

$\mathbf{E}(r, \theta)=\mathbf{E}_{R}(r, \theta)+\mathbf{E}_{\theta}(r, \theta)$,

where

$\mathbf{E}_{R}(r, \theta)=\left(\mathbf{E} \cdot \mathbf{u}_{R}\right) \mathbf{u}_{R}$,

$\mathbf{E}_{\theta}(r, \theta)=\left(\mathbf{E} \cdot \mathbf{u}_{\theta}\right) \mathbf{u}_{\theta}$,

the dot symbolizing the inner product. Each term in Eq. (3) would represent the field at the output of an optical device that only transmits radial or azimuthal components of the input field. Accordingly, $\mathbf{E}_{R}$ is a radially polarized field and $\mathbf{E}_{\theta}$ is azimuthally polarized. At each point of the beam crosssection, the irradiance percentage of the radial component of the field can be evaluated by means of the following ratio:

$\rho_{R}(r, \theta)=\frac{\left\langle\left|\mathbf{E}_{R}(r, \theta)\right|^{2}\right\rangle}{\left\langle|\mathbf{E}(r, \theta)|^{2}\right\rangle}$,

and analogously for the azimuthal component,

$\rho_{\theta}(r, \theta)=\frac{\left\langle\left|\mathbf{E}_{\theta}(r, \theta)\right|^{2}\right\rangle}{\left\langle|\mathbf{E}(r, \theta)|^{2}\right\rangle}$,

where the brackets indicate an ensemble averaging. Taking this into account, the radial and azimuthal polarization content of a non-uniformly totally-polarized field can be characterized by averaging the expressions (5.a) and (5.b) over the region of the beam profile where the irradiance is significant, i.e.,

$$
\begin{aligned}
\tilde{\rho}_{R} & =\frac{\int_{0}^{\infty} \int_{0}^{2 \pi} \rho_{R}(r, \theta)\left\langle|\boldsymbol{E}(r, \theta)|^{2}\right\rangle r \mathrm{~d} r \mathrm{~d} \theta}{\int_{0}^{\infty} \int_{0}^{2 \pi}\left\langle|\boldsymbol{E}(r, \theta)|^{2}\right\rangle r \mathrm{~d} r \mathrm{~d} \theta} \\
& =\frac{\int_{0}^{\infty} \int_{0}^{2 \pi} \rho_{R}(r, \theta) I(r, \theta) r \mathrm{~d} r \mathrm{~d} \theta}{\int_{0}^{\infty} \int_{0}^{2 \pi} I(r, \theta) r \mathrm{~d} r \mathrm{~d} \theta}, \\
\tilde{\rho}_{\theta} & =\frac{\int_{0}^{\infty} \int_{0}^{2 \pi} \rho_{\theta}(r, \theta)\left\langle|\boldsymbol{E}(r, \theta)|^{2}>r \mathrm{~d} r \mathrm{~d} \theta\right.}{\int_{0}^{\infty} \int_{0}^{2 \pi}\left\langle|\boldsymbol{E}(r, \theta)|^{2}\right\rangle r \mathrm{~d} r \mathrm{~d} \theta} \\
& =\frac{\int_{0}^{\infty} \int_{0}^{2 \pi} \rho_{\theta}(r, \theta) I(r, \theta) r \mathrm{~d} r \mathrm{~d} \theta}{\int_{0}^{\infty} \int_{0}^{2 \pi} I(r, \theta) r \mathrm{~d} r \mathrm{~d} \theta},
\end{aligned}
$$

where $I(r, \theta)$ denotes the irradiance at each point of the beam cross-section. The dispersion of the values $\rho_{R}$ and $\rho_{\theta}$ across the beam profile would then be given by

$\sigma_{R}^{2}=\frac{\int_{0}^{\infty} \int_{0}^{2 \pi}\left(\rho_{R}(r, \theta)-\tilde{\rho}_{R}\right)^{2} I(r, \theta) r \mathrm{~d} r \mathrm{~d} \theta}{\int_{0}^{\infty} \int_{0}^{2 \pi} I(r, \theta) r \mathrm{~d} r \mathrm{~d} \theta}$,

$\sigma_{\theta}^{2}=\frac{\int_{0}^{\infty} \int_{0}^{2 \pi}\left(\rho_{\theta}(r, \theta)-\tilde{\rho}_{\theta}\right)^{2} I(r, \theta) r \mathrm{~d} r \mathrm{~d} \theta}{\int_{0}^{\infty} \int_{0}^{2 \pi} I(r, \theta) r \mathrm{~d} r \mathrm{~d} \theta}$.

Of course, the value $\sigma_{R}^{2}$ (or $\sigma_{\theta}^{2}$ ) equal to zero would mean that the beam is uniformly polarized. Also note that $\sigma_{R}^{2}$ and $\sigma_{\theta}^{2}$ represent the variance of $\rho_{R}$ and $\rho_{\theta}$ across the transverse section of the field, with the local value of the irradiance behaving as a density function. Consequently, these parameters $\sigma_{R}^{2}$ and $\sigma_{\theta}^{2}$ globally characterize the uniformity of the radial and azimuthal polarization content over the wavefront.

In addition, it should be noted that both, $\tilde{\rho}_{R}$ and $\tilde{\rho}_{\theta}$, range from 0 to 1 (the value 1 corresponds to a pure radial or azimuthal beam), and satisfy the relation

$\tilde{\rho}_{R}+\tilde{\rho}_{\theta}=1$,

which implies that the radial and the azimuthal polarization content of a beam should be understood as complementary properties. In practice, it would then suffice to determine one of these parameters.

\section{Relations with the Stokes parameters and with the output of radial and azimuthal polarizers}

The parameters defined in the above section can be expressed in terms of the standard Stokes parameters. To show this, note first that (see Eqs. (1) and (4))

$$
\begin{aligned}
& \mathbf{E}_{R}(r, \theta)=\left(\mathbf{E} \cdot \mathbf{u}_{R}\right) \mathbf{u}_{R}=E_{0}(f \cos \theta+g \sin \theta) \\
& \mathbf{E}_{\theta}(r, \theta)=\left(\mathbf{E} \cdot \mathbf{u}_{\theta}\right) \mathbf{u}_{\theta}=E_{0}(-f \sin \theta+g \cos \theta) .
\end{aligned}
$$

Thus we have

$$
\begin{aligned}
\left\langle\left|\mathbf{E}_{R}\right|^{2}\right\rangle= & I_{0}\left\lfloor\cos ^{2} \theta\left\langle|f|^{2}\right\rangle+\sin ^{2} \theta\left\langle|g|^{2}\right\rangle\right. \\
& \left.+2 \sin \theta \cos \theta \operatorname{Re}\left\{\left\langle f^{*} g\right\rangle\right\}\right\rfloor \\
\left\langle\left|\mathbf{E}_{\theta}\right|^{2}\right\rangle= & I_{0}\left\lfloor\sin ^{2} \theta\left\langle|f|^{2}\right\rangle+\cos ^{2} \theta\left\langle|g|^{2}\right\rangle\right. \\
& \left.-2 \sin \theta \cos \theta \operatorname{Re}\left\{\left\langle f^{*} g\right\rangle\right\}\right\rfloor
\end{aligned}
$$

where $I_{0}=\left\langle\left|E_{0}\right|^{2}\right\rangle$. Taking this into account, we get

$$
\begin{aligned}
\left\langle\left|\mathbf{E}_{R}\right|^{2}\right\rangle & =\frac{1}{2} s_{0}+\frac{\cos 2 \theta}{2} s_{1}+\frac{\sin 2 \theta}{2} s_{2}, \\
\left\langle\left|\mathbf{E}_{\theta}\right|^{2}\right\rangle & =\frac{1}{2} s_{0}-\frac{\cos 2 \theta}{2} s_{1}-\frac{\sin 2 \theta}{2} s_{2},
\end{aligned}
$$

where $s_{0}, s_{1}$ and $s_{2}$ are the conventional Stokes parameters determined at each point $(r, \theta)$ of the transverse beam profile. From the definition of $\tilde{\rho}_{R}$ and $\tilde{\rho}_{\theta}$ we finally obtain

$$
\begin{aligned}
\tilde{\rho}_{R}= & \frac{1}{2}+\frac{1}{2 P} \int_{0}^{\infty} \int_{0}^{2 \pi}\left[\cos (2 \theta) s_{1}(r, \theta)\right. \\
& \left.+\sin (2 \theta) s_{2}(r, \theta)\right] r \mathrm{~d} r \mathrm{~d} \theta,
\end{aligned}
$$




$$
\begin{aligned}
\tilde{\rho}_{\theta}= & \frac{1}{2}-\frac{1}{2 P} \int_{0}^{\infty} \int_{0}^{2 \pi}\left[\cos (2 \theta) s_{1}(r, \theta)\right. \\
& \left.+\sin (2 \theta) s_{2}(r, \theta)\right] r \mathrm{~d} r \mathrm{~d} \theta,
\end{aligned}
$$

where $P=\int_{0}^{\infty} \int_{0}^{2 \pi} s_{0}(r, \theta) r \mathrm{~d} r \mathrm{~d} \theta$ denotes the total power of the beam. Remember that, to measure the above Stokes parameters at each point, we can handle, for example, a CCD camera placed at the observation plane, together with a polarizer at different orientations, and use the wellknown expressions:

$s_{0}(r, \theta)=I_{0}(r, \theta)+I_{90}(r, \theta)$,
$s_{1}(r, \theta)=I_{0}(r, \theta)-I_{90}(r, \theta)$,
$s_{2}(r, \theta)=I_{45}(r, \theta)-I_{135}(r, \theta)$.

In these equations, the subscripts indicate the angle that the transmission axis of the polarizer makes with the $x$-axis.

As is quite apparent from Eqs. (12), $\tilde{\rho}_{R}$ and $\tilde{\rho}_{\theta}$ do not depend on the local Stokes parameter $s_{3}$, so that no information about $s_{3}$ is required to characterize the radial or azimuthal polarization content of a beam. This behavior should physically be expected because $s_{3}$ equals zero for any radially (or azimuthally) polarized field (according with the definitions (4.a) and (4.b)). Moreover, it is interesting to note that this also applies for the particular case of a radially polarized beam with vortex wavefront (see, for example, Ref. [26] and references therein). In such case, the global phase factor that defines the topological charge does not alter the relative phase term between the transverse components and, consequently, the parameter $s_{3}$ remains equal to zero. One would naively expect this independence on $s_{3}$ because this Stokes parameter provides information about the "circularity" of the polarization state (in fact, $s_{3}$ reaches its maximum value for a circularly polarized beam), and the polarization of a pure radially (or azimuthally) polarized field is strictly linear throughout the beam cross-section.

Instead of measuring the local values of the Stokes parameters, it would be of practical use to determine the parameters $\tilde{\rho}_{R}$ and $\tilde{\rho}_{\theta}$ from the integrated data over the full detection area. In such a case, we would not need to perform detailed (local) detections but a global single irradiance measurement. This can be achieved in a simple way by using special (commercially available) devices, namely, either a radial or an azimuthal polarizer. In fact, it can be shown at once that

$\tilde{\rho}_{R}=\frac{1}{P} \int_{0}^{\infty} \int_{0}^{2 \pi}\left\langle\left|P_{R} \mathbf{E}(r, \theta)\right|^{2}\right\rangle r \mathrm{~d} r \mathrm{~d} \theta$

where $P_{R} \mathbf{E}(r, \theta)=\left(\mathbf{E} \cdot \mathbf{u}_{R}\right) \mathbf{u}_{R}$ would represent the (pointdependent) output of the polarizer. Accordingly, the integral appearing in the numerator of Eq. (14) would just give the global irradiance (integrated throughout the wavefront) collected at the output of the polarizer. It is important to remark that, in order to implement the operation expressed by Eq. (14), the polarizer should eliminate the azimuthal component $\mathbf{E}_{\theta}$ of the input field (see Eq. (3)).
Consequently, a radial dichroic polarizer should be employed (each tiny area of such a polarizer would behave like a linear polarizer with the transmission axis in the radial direction).

In an analogous way, $\tilde{\rho}_{\theta}$ can be written in the form

$\tilde{\rho}_{\theta}=\frac{1}{P} \int_{0}^{\infty} \int_{0}^{2 \pi}\left\langle\left|P_{\theta} \mathbf{E}(r, \theta)\right|^{2}\right\rangle r \mathrm{~d} r \mathrm{~d} \theta$

where now $P_{\theta} \mathbf{E}(r, \theta)=\left(\mathbf{E} \cdot \mathbf{u}_{\theta}\right) \mathbf{u}_{\theta}$ would denote the output of an azimuthal dichroic polarizer. This kind of polarizer is well known in the literature, used as polarization axis finder. Of course, both parameters $\tilde{\rho}_{R}$ and $\tilde{\rho}_{\theta}$ are linked by Eq. (8).

\section{Measurability of the overall parameters}

The measurability of parameters $\tilde{\rho}_{R}$ and $\tilde{\rho}_{\theta}$ has been checked by testing two optical devices, namely, a conventional linear polarizer and a liquid-crystal polarization converter, both from commercial companies.

The Arcoptix polarization converter used in the experiments is based on a nematic liquid-crystal cell, which can be switched to obtain either radial or azimuthal polarization distribution from a linearly polarized input beam [5].

In all the measurements, $\tilde{\rho}_{R}$ and $\tilde{\rho}_{\theta}$ were found by determining the local Stokes parameters (cf. Eq. (12.a) and (12.b)). The images were taken with a CCD camera model Pulnix TM-765 and a laser beam analyzer from Spiricon. The set-up is represented in Figs. 1a and b.

When we consider the linear polarizer, its extinction ratio is (according with the manufacturer specifications) $T 1 / T 2=250$ and the light source used in the measurements was a linearly polarized Gaussian beam emitted by a Spectra Physics He-Ne laser device. The experimental result was $\tilde{\rho}_{R}=\tilde{\rho}_{\theta}=0.499$ (both parameters were calculated independently), showing a good agreement with the expected theoretical value $\tilde{\rho}_{R}=\tilde{\rho}_{\theta}=0.5$.

In the other test, when the $\mathrm{He}-\mathrm{Ne}$ laser beam impinges over the polarization converted switched to get radial polarization, we obtained for the output beam $\tilde{\rho}_{R}=0.986$ and $\tilde{\rho}_{\theta}=0.014$, with $\sigma_{R}^{2}=\sigma_{\theta}^{2}=0.009$, showing an excellent agreement with the values $\tilde{\rho}_{R}=1, \quad \tilde{\rho}_{\theta}=0, \sigma_{R}^{2}=\sigma_{\theta}^{2}=0$ associated to a pure radially polarized beam. Fig. 2 gives the irradiance distributions at the output of the polarization converter, driven in the radial mode. In this figure, close to the center of symmetry we have a small (about $400 \mu \mathrm{m}$ ) region (a central hole) with undefined liquid-crystal orientation. This arises from the construction principle of the cell (for details, see, for example, Ref. [5]). The influence of this center on the values of parameters $\tilde{\rho}_{R}$ and $\tilde{\rho}_{\theta}$ is, however, negligible for the propagation distances handled in the experiments.

Fig. 3 plots the irradiance distributions at the output of the linear polarizer $P_{2}$ for different orientations of its transmittance axis (the angles $0^{\circ}, 90^{\circ}, 45^{\circ}$ and $135^{\circ}$ correspond to the values required to measure the Stokes parameters). In figures, the number and position of the lobes agree with 
a

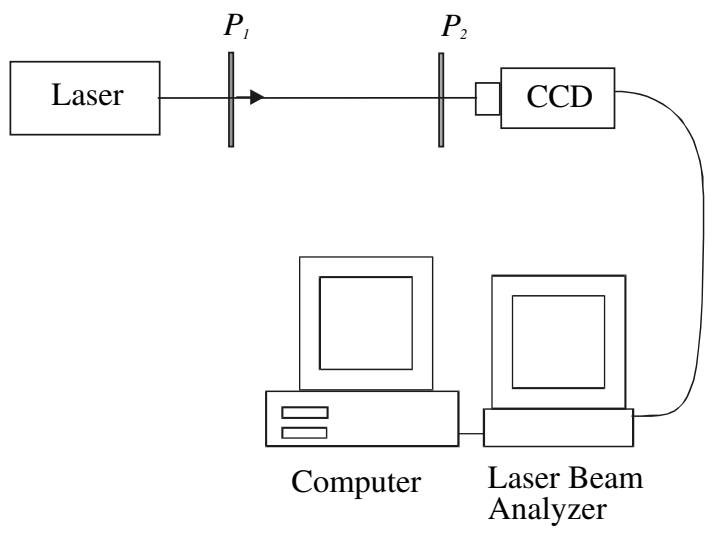

b

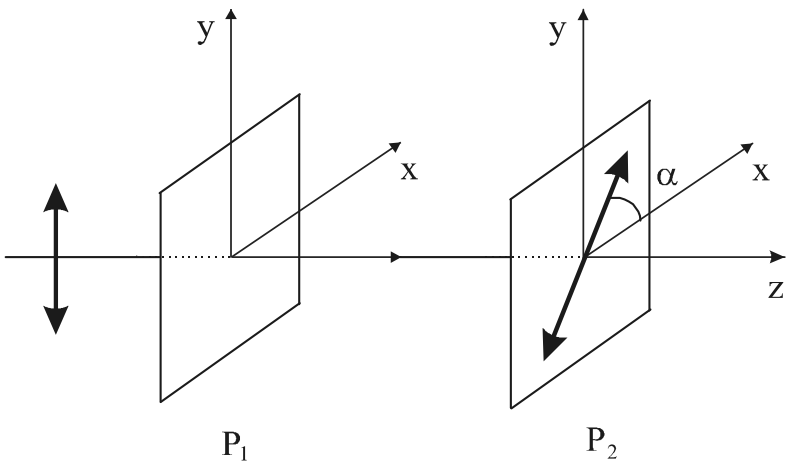

Fig. 1. (a) Experimental set-up for measuring the proposed parameters. $P_{1}$ represents either the linear polarizer or the liquid-crystal polarization converter tested in the experiments, and $P_{2}$ denotes the linear polarizer used to determine the Stokes parameters. (b) Cartesian reference axes used in the experiments. In all the cases, the beam emerging from the laser cavity is linearly polarized along the $y$-axis. To measure the Stokes parameters, the transmission axis of the polarizer $P_{2}$ (analizer) makes angles $\alpha\left(0^{\circ}, 90^{\circ}, 45^{\circ}, 135^{\circ}\right)$ with respect to the $x$-axis. The $z$-axis is the propagation direction of the beam.

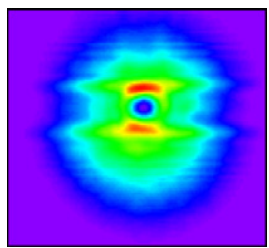

Fig. 2. Irradiance distribution (pseudocolour) after the polarization converter in the radial mode (see the main text), without $P_{2}$.

the theoretical predictions for the beam emerging from the radial polarization converter.

The experiments were also performed in the azimuthal mode of the liquid-crystal device. We obtained $\tilde{\rho}_{R}=0.012$ and $\tilde{\rho}_{\theta}=0.988$, with $\sigma_{R}^{2}=\sigma_{\theta}^{2}=0.007$. This shows an excellent agreement with the values $\tilde{\rho}_{R}=0$, $\tilde{\rho}_{\theta}=1, \sigma_{R}^{2}=\sigma_{\theta}^{2}=0$ that characterize a pure azimuthally polarized beam. For completeness, Fig. 4 plots the irradiance profile (quite similar to that of Fig. 2) at the output of the polarization converter, and Fig. 5 gives the irradiance distributions at the output of $P_{2}$, driven in the azi-

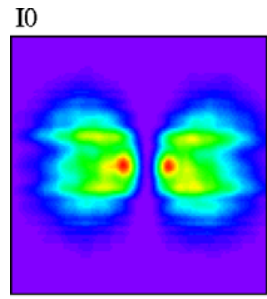

190
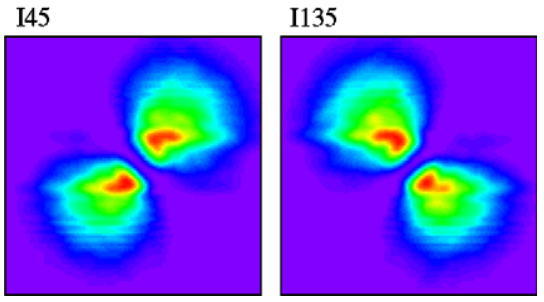

Fig. 3. Irradiance distributions after $P_{2}$ for the orientations of its transmission axis $\left(0^{\circ}, 90^{\circ}, 45^{\circ}\right.$ and $\left.135^{\circ}\right)$ used to measure the local Stokes parameters $s_{0}, s_{1}$ and $s_{2}$ of the beam emerging from the polarization converter operating in the radial mode.

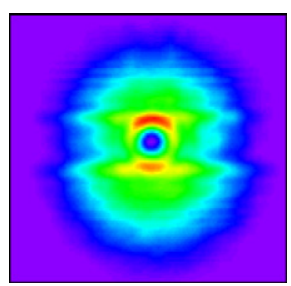

Fig. 4. Irradiance distribution after the polarization converter in the azimuthal mode (see the main text), without $P_{2}$.
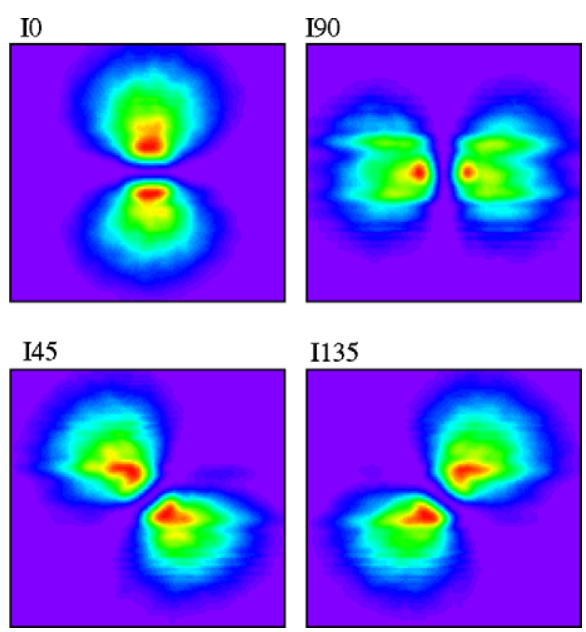

Fig. 5. Irradiance distributions after $P_{2}$ for the orientations of its transmission axis $\left(0^{\circ}, 90^{\circ}, 45^{\circ}\right.$ and $\left.135^{\circ}\right)$ used to measure the local Stokes parameters $s_{0}, s_{1}$ and $s_{2}$ of the beam emerging from the polarization converter operating in the azimuthal mode.

muthal mode. All the above results confirm the measurability of the proposed parameters.

In the experiments, special care was taken to avoid misalignments between the optical components of the system and the laser beam, which should cross through the centre 
of the devices (the spatial inhomogeneities could produce harmful effects when the components rotate).

In the above measurements, it was first confirmed that the output beams were totally polarized throughout the beam profile. To do this, we measured the so-called weighted degree of polarization, $\tilde{P}$, and its variance, $\tilde{\sigma}_{p}^{2}$, namely [22],

$\tilde{P}=\frac{\int I(r, \theta) P(r, \theta) r \mathrm{~d} r \mathrm{~d} \theta}{\int I(r, \theta) r \mathrm{~d} r \mathrm{~d} \theta}$,

$\tilde{\sigma}_{p}^{2}=\frac{\int I(r, \theta)[P(r, \theta)-\tilde{P}]^{2} r \mathrm{~d} r \mathrm{~d} \theta}{\int I(r, \theta) r \mathrm{~d} r \mathrm{~d} \theta}$,

where $P(r, \theta)$ represents the well-known local degree of polarization [21]. Note that, for this kind of measurements, a quarter-wave plate is also required in the experimental set-up. The transmittance of this plate was $T=0.98$. It should be recalled that the value $\tilde{P}=1$ indicates that we handle a pure totally-polarized beam. After the linear polarizer tested in the experiment, we got $\tilde{P}=1.014$ and $\tilde{\sigma}_{p}^{2}=0.001$. This means that the output field is totally polarized everywhere. The same conclusion arises for the beam emerging from the polarization converter $\left(\tilde{P}=1.08, \tilde{\sigma}_{p}^{2}=0.08\right.$ in the radial case, and $\tilde{P}=1.09$, $\tilde{\sigma}_{p}^{2}=0.09$ in the azimuthal one).

\section{Conclusions}

The radial and azimuthal polarization content of a nonuniformly totally polarized beam can be characterized by means of two overall parameters, $\tilde{\rho}_{R}$ and $\tilde{\rho}_{\theta}$, defined as certain averages over the region of the beam profile where the irradiance is significant. From an analytical and experimental point of view, these figures of merit have been obtained in terms of the Stokes parameters $s_{0}, s_{1}$ and $s_{2}$. Furthermore, to measure $\tilde{\rho}_{R}$ and $\tilde{\rho}_{\theta}$, it has been shown that it is not necessary to determine local values. Instead, the proposed parameters can be experimentally inferred from the data, integrated over the beam cross-section, at the output of radial and azimuthal dichroic polarizers.

The spatial uniformity, across the beam profile, of the polarization-content distributions can be analytical and experimentally evaluated in terms of the respective variances, $\sigma_{R}^{2}$ and $\sigma_{\theta}^{2}$. Finally, the measurability of the above parameters has been tested from the output of commercially available devices.

\section{Acknowledgments}

This work has been supported by the Ministerio de Educación y Ciencia of Spain, under project FIS2004-1900, and by the Universidad Complutense-Comunidad de Madrid, project No. 910335 (2007). We thank Dr. Julio Serna for his valuable suggestions.

\section{References}

[1] S.C. Tidwell, D.H. Ford, W.D. Kimura, Appl. Opt. 29 (1990) 2234.

[2] T. Erdogan, D.G. Hall, J. Appl. Phys. 68 (1990) 1435.

[3] T. Erdogan, O. King, G.W. Wicks, D.G. Hall, E. Anderson, M.J. Rooks, Appl. Phys. Lett. 60 (1992) 1921.

[4] S.C. Tidwell, G.H. Kim, W.D. Kimura, Appl. Opt. 32 (1993) 5222.

[5] M. Stalder, M. Schadt, Opt. Lett. 21 (1996) 1948.

[6] V.G. Niziev, A.V. Nesterov, J. Phys. D 32 (1999) 1455.

[7] S. Nolte, C. Momma, G. Kamlage, A. Ostendorf, C. Fallnich, F. von Alvensleben, H. Welling, Appl. Phys. A 68 (1999) 563.

[8] I. Freund, Opt. Commun. 199 (2001) 47.

[9] L. Novotny, M.R. Beversluis, K.S. Youngworth, T.G. Brown, Phys. Rev. Lett. 86 (2001) 5251.

[10] G. Piquero, F. Gori, P. Romanini, M. Santarsiero, R. Borghi, A. Mondello, Opt. Commun. 208 (2002) 9.

[11] J. Tervo, J. Opt. Soc. Am. A 20 (2003) 1974.

[12] R. Dorn, S. Quabis, G. Leuchs, Phys. Rev. Lett. 91 (2003) 233901/1.

[13] I. Moshe, S. Jackel, A. Meir, Opt. Lett. 28 (2003) 807.

[14] C.J.R. Sheppard, A. Choudhury, Appl. Opt. 43 (2004) 4322.

[15] M.J. Padgett, J. Opt. A: Pure Appl. Opt. 6 (2004) 263.

[16] M. Erdelyi, Z. Bor, J. Opt. A: Pure Appl. Opt. 8 (2006) 737.

[17] K. Yonezawa, Y. Kozawa, S. Sato, Opt. Lett. 31 (2006) 2151.

[18] G. Machavariani, Y. Lumer, I. Moshe, A. Meir, S. Jackel, Opt. Lett. 32 (2007) 1468.

[19] D. Deng, Q. Guo, Opt. Lett. 32 (2007) 2711.

[20] M. Born, E. Wolf, Principles of Optics, Cambridge University Press, Cambridge, 1999.

[21] C. Brosseau, Fundamentals of Polarized Light, Wiley, New York, 1998.

[22] J.M. Movilla, G. Piquero, R. Martínez-Herrero, P.M. Mejías, Opt Commun. 149 (1998) 230.

[23] G. Piquero, J.M. Movilla, R. Martínez-Herrero, P.M. Mejías, Opt. Quantum Electron. 31 (1999) 223.

[24] P.M. Mejías, R. Martínez-Herrero, G. Piquero, J.M. Movilla, Prog. Quantum Electron. 26 (2002) 65.

[25] R. Martínez-Herrero, P.M. Mejías, G. Piquero, Opt. Commun. 265 (2006) 6.

[26] G. Machavariani, Y. Lumer, I. Moshe, S. Jackel, Opt. Commun. 271 (2007) 190 